\title{
The Discourse Analysis and Translation Method of Diplomatic Speeches in Chinese and English from the Perspective of the Appraisal System -a Case study of President Xi visiting England in 2015
}

\author{
Xiao Zhang \\ Col. of Humanities and Law, North China Univ. of Tech., 100144, Beijing, China \\ uphere99@163.com
}

\begin{abstract}
Keywords: Appraisal system; Diplomatic speeches; Discourse analysis; Translation between English and Chinese
\end{abstract}

\begin{abstract}
This article has applied the appraisal system to discourse analysis of diplomatic speeches in both Chinese and English. With the help of statistical data, it can be generalized that such bilingual texts have some stylistic similarities as well as some differences and these are inspiring in the translation of this genre of texts. Proper translation methods for such texts could be chosen according to the appraisal system, which is an important and valuable system.
\end{abstract}

\section{Introduction}

In the late 1950s, an English linguist M.A.K. Halliday established systemic-functional grammar. This form, compared with traditional grammar, structural grammar and transformational-generative grammar, emphasizes more on the influence of discourse and context on language. Systemicfunctional grammar can be divided into two parts - systemic grammar and functional grammar. In the latter part, three metafunctions have been generalized by Halliday, namely, ideational function, interpersonal function and textual function, for better explanation of language utilization from three different perspectives. "However, in terms of observing the opinions and stances of the writer/reader or the speaker/listener through language, systematic-functional grammar is still not fully developed.'[1] Then in the 1990s, J.R. Martin and P. R. R. White jointly developed the appraisal system.

First of all, appraisal can be regarded as a dynamic mechanism consisting of three main subsystems - attitude, engagement and graduation. Secondly, each sub-system forms a separate system while it is subject to the upper level. Thus a discourse can be analyzed in detail from various perspectives. The application of appraisal has an extensive influence and is worth promoting.

Published papers by far have mostly focused on either theory introduction or the application of appraisal to discourse analysis. For the latter part, discourses are mainly concentrated on areas such as political speeches, public speeches and presidential inaugural addresses, most of which are in English. The aim of researchers, on one side, is to conduct detailed analysis of those discourses so as to help readers/listeners get a better understanding, and then magage to communicate. On the other side, we can learn from speakers/ writers how to communicate well by using the appraisal system appropriately.

It's clear that Chinese or bilingual discourses are seldom seen in these application. One of the reason may be that the appraisal system was developed for English at first, and it's harder to adapt it to ideogram. However, it's much easier to apply appraisal to English-Chinese translation. We'll illustrate this in this article later.

\section{An Introduction of the Appraisal System}

Appraisal is to evaluate the standpoint, opinion and attitude of language users towards some situation through analysis of language. In the three main subsystems--attitude, engagement and graduation--attitude is the core of the whole system. 
Attitude includes "the assessment of human feelings, of human characters and behaviors, of the value of things", referring to affection, judgment and appreciation respectively.[2] Each subsystem of attitude has its own appraisal meaning, both positively and negatively.[3] Among these three subsystems, affection is the core of attitude. Thus a clear core clue of thought can be grasped: appraisal-attitude - affection, which is the most basic, most important and most wild-spread application of appraisal in discourse analysis. This thread can serve as a direction when we try to read the potential and implied attitude of writers/ speakers between the lines (whether he/ she is for, against or neutral on something), and then adjust the strategies of communication to achieve the purpose of intercourse.

Engagement, divided into monogloss and heterogloss, focuses on the way by which writers/ speakers express the attitude, point or opinion. "Engagement means the engagement of attitude from the perspective of linguistics. When people express their attitudes, they would speak in an either direct or indirect way." [4] Through the following four ways, disclaim, proclaim, entertain and attribute, language users can express their views either directly or indirectly.

Graduation "mainly uses ways such as strengthening, comparison, the superlative degree, repetition, parallelism and alliteration to intensify force, thus making attitude more appealing and language more powerful and infectious".[5] Thus it can be seen that the demonstration of graduation is mainly including the above-mentioned ways as well as all kinds of character and phonological features. [6]

Graduation also forms a separate subsystem, consisting of two sub-categories of force and focus. Force, which can be divided into raise and lower, is used to increase or decrease impression and verbal capacity. Words like slightly, somewhat and so on have a lower force; while words suck as completely, extremely and fantastic have a higher force. [1] Focus can be divided into sharpen and soften, with a role of making the semantic meaning clearer or vaguer. Expressions suck as "I am feeling kind of weird" have a vaguer meaning (than "I am feeling weird"); while "a true friend of his" has a much clearer meaning (than "a friend of his"). [1]

Among the above subsystems, some can still be divided into more detailed subsystems. We won't mention them because they are irrelevant to our analysis here.

\section{An Introduction of Diplomatic Speeches}

Diplomatic speeches "can be seen as the dictation of written language".[7] This is such a style that it discusses major issues and realistic problems at home and abroad from the perspective of political views. Backgrounds such as the social culture, tradition, history and ideology represented by speakers can be conveyed.[8] In diplomatic speeches, speakers would resort to direct or indirect ways to reflect or imply his/her underlying meaning, thus revealing his/her true attitude and opinions. These strategies used are the embodiment of appraisal.

As for audiences or listeners, that the stylistic features of diplomatic speeches-both concise and deep - as well as the use of many rhetoric makes it harder to understand the core thought of these speeches. They need a scientific and systematic system to get the picture of these discourses.

In 2015, President Xi Jinping of China visited England and both leaders delivered speeches during the dinner. This article aims to conduct discourse analysis of the two discourses with the help of the appraisal system, hoping to appreciate the parallel texts and get inspired when doing English-Chinese translation of this kind of style.

\section{Contrast and Comparison of the Bilingual Discourses}

Method. First of all, different expressions are categorized under different sub-categories of the appraisal system. By a statistical approach, we will get an approximate rate of each sub-category. After observing similarities and differences among these statistics, we can study how to grasp the discourses correctly and translate them appropriately. 
Attitude. Three similarities are shown in graph one: first, most of the expressions are positive in both discourses. Second, in the three subcategories, appreciation accounts for a larger proportion. Third, the proportion of each category in both discourses is similar.

Table 1 Contrast and comparison of attitude in bilingual discourses

\begin{tabular}{|c|c|c|c|c|c|c|}
\hline \multirow{2}{*}{ Discourse } & \multicolumn{2}{|c|}{ Affection } & \multicolumn{2}{c|}{ Judgement } & \multicolumn{2}{c|}{ Appreciation } \\
\cline { 2 - 7 } Content & positive & negative & positive & negative & positive & negative \\
\hline Chinese & $2(6 \%)$ & 0 & $15(44 \%)$ & 0 & $14(41 \%)$ & $3(9 \%)$ \\
\hline English & $4(11 \%)$ & 0 & $15(43 \%)$ & 0 & $7(20 \%)$ & $9(26 \%)$ \\
\hline
\end{tabular}

The reason why both discourses focus on positive expressions and have a similar proportion in every category lies in their stylistic features. Diplomatic speeches, to some extent, belong to a high context culture, and politeness is attached to great importance.

Graduation. There is a distinct difference in graph two. Chinese discourse emphasizes on vague expressions while English discourse values clear expressions. This has some connection with the cultural background. Chinese people like to express ambiguously, perhaps influenced by the ancient moderate attitude. Englishmen, or western people, prefer to use direct and clear expressions, a demonstration of their low context culture.

Table 2 Contrast and comparison of graduation in bilingual discourses

\begin{tabular}{|c|c|c|c|c|c|}
\hline \multirow{2}{*}{$\begin{array}{c}\text { Discourse } \\
\text { Content }\end{array}$} & \multirow{2}{*}{ Number } & \multicolumn{2}{|c|}{ Force } & \multicolumn{2}{c|}{ Focus } \\
\cline { 3 - 6 } & & Raise & Lower & Sharpen & Soften \\
\hline Chinese & 13 & $5(38 \%)$ & 0 & $1(8 \%)$ & $7(54 \%)$ \\
\hline English & 22 & $19(86 \%)$ & 0 & $1(5 \%)$ & $2(9 \%)$ \\
\hline
\end{tabular}

Engagement. From the graph, we can see that Chinese are used to expressing facts instead of personal feelings, especially avoiding speaking in the first-person viewpoint, which embodies their implicit character. In the English discourse, both ways of engagement appear. Monogloss can show the credibility and heterogloss can show the objectivity.

Table 3 Contrast and comparison of engagement in bilingual discourses

\begin{tabular}{|c|c|c|c|c|}
\hline Discourse & \multirow{2}{*}{ Number } & \multicolumn{3}{|c|}{ Content } \\
\hline Content & 4 & \multicolumn{2}{|c|}{$\mathrm{I}(3)$} & $\mathrm{We}(1)$ \\
\hline Eninese & 9 & Prince Philip and I (2) & $\mathrm{We}(1)$ & $\mathrm{I}(4)$ \\
\hline
\end{tabular}

From the Perspective of Parts of Speech. As shown in graph four, in the area of judgments, appreciation and graduation, the distribution of part of speeches in the two discourses is different. Taking graduation for example, the Chinese discourse mainly focuses on adjective and adverb, and the English discourse would like to use all the four parts of speeches. This difference can help translators a lot during English-Chinese translation, which will be mentioned later.

\section{Conclusion}

By far, we have found some similarities and differences during the above contrast and comparison, two points of which are valuable.

First, the distribution of appraisal words in both discourses witnesses a similar trend. Positive words account for a larger proportion, mainly focusing on appreciation and judgment. This phenomenon has a lot to do with the aim and register of communication.

Second, the distribution of parts of speech sees some difference in both discourses. 
Similarity proves that this analysis has some meaning and difference can tell us what the meaning is or in which way this analysis can be used.

Table 4 Contrast and comparison of two main subsystems in bilingual discourses from the perspective of parts of speech

\begin{tabular}{|c|c|c|c|c|c|c|c|c|c|}
\hline \multirow{2}{*}{ Content } & \multicolumn{2}{c|}{ Discourse } & \multicolumn{2}{c|}{ Noun } & \multicolumn{2}{c|}{ Adjective } & \multicolumn{2}{c|}{ Adverb } & \multicolumn{2}{c|}{ Verb } \\
\cline { 3 - 10 } \multicolumn{2}{|c|}{ Content } & Chi. & Eng. & Chi. & Eng. & Chi. & Eng. & Chi. & Eng. \\
\hline \multirow{3}{*}{ Attitude } & Affection & 1 & 1 & 1 & 3 & $/$ & $/$ & $/$ & $/$ \\
\cline { 2 - 10 } & Judgement & 4 & 9 & 4 & 6 & 4 & $/$ & 3 & $/$ \\
\cline { 2 - 10 } & Appreciation & 13 & 10 & 2 & 6 & $/$ & $/$ & 2 & $/$ \\
\hline \multicolumn{2}{|c|}{ Graduation } & $/$ & 2 & 12 & 14 & 1 & 5 & $/$ & 1 \\
\hline \multicolumn{2}{|c|}{ Total } & 18 & 22 & 19 & 29 & 5 & 5 & 5 & 1 \\
\hline
\end{tabular}

\section{Inspirations to English-Chinese Translation}

The application of the appraisal system in translation mainly focuses on the extent to which translators can understand the source text and the translation criteria. [9] Equivalence is one criterion to evaluate one translation, and the equivalence of appraisal meaning is included. In many situations, to translate the semantic and pragmatic meaning of the source text means to translate the appraisal meaning well. The cognation of the world and personal experiences of translators are factors affecting the understanding of source texts.

From the above contrast and comparison, it is clear that diplomatic speeches do have some characteristics, which need to be conveyed to the readers. For example, wording should focus more on positive than negative ones.

The difference told in the summary in 3.5 shows that translators can choose to change the parts of speech in order to achieve the equivalence of stylistic features.

Look at the following example, selected from the English discourse:

This global partnership is supported by an expanding network of ...which are essential in building mutual understanding and friendship...

Analysis: the word "essential" means "extremely important, absolute necessary". In Chinese, we have an equivalence of "important", and many dictionaries will give the same translation of the word "essential" as "important", so it's vague about the difference of the two words if we understand them from Chinese. This causes that some translators would just translate "essential" as "important" without noticing the difference. Here the difference lies in their graduation. As mentioned above, in Chinese, adjective and adverb are widest use in expressing graduation, so we can choose the "addition strategy" by adding an adverb, such as the equivalence of "very, extremely" in Chinese to achieve the equivalence.

Tips: The meaning of one English word may be expressed by two or more Chinese words. Translators need to get a good knowledge of the difference of English words, especially synonyms.

By the way, choosing translation strategies from the perspective of the appraisal system could also provide inspiring ideas when translating other kinds of source texts.

\section{Conclusion}

The appraisal system, since it was developed and spread, has seen wild application in discourse analysis. The appraisal theory provides a pretty systematic method to guide readers/ listeners to get a better understanding of the true meaning of what the writers/ speakers express, so as to achieve effective communication. Nowadays, this theory has offered many inspirations in areas such as stylistics, translation, writing and political adresses. 
Indeed, the appraisal system does have some flaws. The system itself should be improved for it is aimed at English and needs adjustment when applied to other languages that are not belong to phonograph. When sorting out which word belongs to which subsystem, the criterion is subjective.

The appraisal system can assess the ideology of language users mostly through evaluative lexis. It has a broad application because of revealing things that cannot, or hardly, do by other linguistics branches. It's dependence on lexis is also its shortcoming.[10] When it comes to semantic and contextual levels, the appraisal theory cannot do much.

Though there exist some flaws, the appraisal system still plays a vast and incredible role when conducting discourse analysis, and it's one comparatively perfect and systematic method. This theory can do detailed analysis and help readers/ listeners get a more accurate comprehension of the discourse, even can provide inspirations for translation and linguistics. The appraisal system is a good tool for the study and learn of foreign language.

\section{References}

[1] Liu Runqing. Schools of Linguistics, Beijing: Foreign Language Teaching and Research Press, 2013.

[2] Yu Tao. A study of textual equivalence translation from the perspective of the appraisal theory [J]. Journal of Xuzhou Normal University (Philosophy and Social Sciences Edition), 2011 (06): 66-68.

[3] Guan Shuhong, Wang Yali and Luo Lingjuan. An Analysis on Political Speech from the Perspective of Appraisal System [J]. Journal of East China Jiaotong University, 2006(06): 7478.

[4] Wang Zhenhua and Lu Yang. A reflection on engagement systems[J]. Foreign Language Research, 2010(03): 51-56.

[5] Chen Songyun. An analysis of Obama's speech on the death of Bin Laden from the perspective of the appraisal theory [J]. Overseas English. 2011 (11): 323-327.

[6] J.R. Martin and P.R.R. White. The Language of Evaluation - Appraisal in English, London: Palgrave Macmillan, 2005.

[7] Zhong Lili. On Lexical Choice in Political Speeches from the Perspective of Appraisal System [J].Journal of Shaoxing University, 2005(01): 54-57.

[8] Chen Yi. The application of the appraisal theory in diplomatic speeches [J]. Journal of Chongqing University of Science and Technology (Social Sciences Edition). 2011 (06): 117118,129 .

[9] Liu Shizhu. The development of the appraisal system in China [J]. Foreign Languages and Their Teaching, 2010 (05): 33-37.

[10]Wang Zhenhua. Appraisal systems and their operation: a new development in the systemic functional linguistics [J]. Journal of Foreign Languages, 2001 (06): 13-20. 